\title{
Effects of salidroside on exhaustive exercise-induced oxidative stress in rats
}

\author{
JIANSHENG XU ${ }^{1}$ and YING $\mathrm{LI}^{2}$ \\ ${ }^{1}$ Department of Physical Education, Tianjin University, Tianjin 300072; \\ ${ }^{2}$ Department of Physical Education, Tianjin Agricultural University, Tianjin 300084, P.R. China
}

Received April 15, 2012; Accepted August 16, 2012

DOI: $10.3892 / \mathrm{mmr} .2012 .1060$

\begin{abstract}
Intense exercise increases oxygen consumption and may produce an imbalance between reactive oxygen species (ROS) and antioxidants, inducing oxidative stress as a result of increased ROS production. Exogenous antioxidants may prevent oxidative damages since they are able to detoxify certain peroxides by scavenging the ROS produced during exercise. The aim of this study was to evaluate the effects of salidroside on exhaustive exercise-induced oxidative stress in rats. A total of 40 animals were randomly divided into four groups of ten rats each: control (C), low-dose salidroside-treated (LT), middle-dose salidroside-treated (MT) and high-dose salidroside-treated (HT) groups. The rats in the treated groups received salidroside $(25,50$ and $100 \mathrm{mg} / \mathrm{kg}$, respectively) intragastrically (ig) and the rats in the control group received drinking water ig for 4 weeks. After 4 weeks, the rats performed an exhaustive swimming exercise and exhaustive swimming times were recorded. The malondialdehyde (MDA), catalase (CAT), superoxide dismutase (SOD), glutathione peroxidase (GSH-Px) and glycogen levels in the liver tissues of the rats were measured. The data revealed that salidroside was able to elevate the exercise tolerance and increase the liver glycogen levels of the rats following exhaustive exercise. Salidroside was also able to reduce MDA levels and enhance the activities of antioxidant enzymes (CAT, SOD and GSH-Px) in the liver tissues of the rats. The results from this study indicate that salidroside is effective in the prevention of oxidative stress following exhaustive exercise.
\end{abstract}

\section{Introduction}

Cells continuously produce free radicals and reactive oxygen species (ROS) as part of their metabolic processes. These free radicals are neutralized by an elaborate antioxidant

Correspondence to: Dr Jiansheng Xu, Department of Physical Education, Tianjin University, 92 Weijin Road, Nankai District, Tianjin 300072, P.R. China

E-mail: jishxu@163.com

Key words: salidroside, exhaustive exercise, oxidative stress, rats defense system consisting of enzymes, including catalase (CAT), superoxide dismutase (SOD) and glutathione peroxidase (GSH-Px), and numerous non-enzymatic antioxidants, including vitamin $\mathrm{E}$, vitamin $\mathrm{A}$, vitamin $\mathrm{C}$, glutathione and uric acid $(1,2)$. Intense exercise increases oxygen consumption and may produce an imbalance between ROS and antioxidant levels, inducing oxidative stress as a result of increased ROS production (3). Indeed, exercise-induced oxidative stress leads to the destruction of tissue and cell macromolecules, including lipids, proteins and nucleic acids (4-6). It has also been suggested that exercise-induced oxidative stress may be associated with muscle fatigue, muscle damage and a decrease in physical performance (7-9). Antioxidants are substances that help to reduce the severity of oxidative stress, either by forming a less active radical or by quenching the reaction. It has been demonstrated that exogenous antioxidants may prevent oxidative damage since they are able to detoxify certain peroxides by scavenging the ROS produced during exhaustive exercise (10).

Rhodiola rosea ("golden root" or "Arctic root") is widely distributed at high altitudes in the Arctic and in mountainous regions throughout Europe and Asia (11). It has been widely used as a hemostatic, antitussive, tonic and endermic liniment for the treatment of burns and contusions in traditional Chinese medicine (12). Salidroside, 2-(4-hydroxyphenyl)ethyl $\beta$-D-glucopyranoside, the main active compound of Rhodiola rose $a$, is reported to exert antiviral, antidiabetic, antifatigue, antiaging, neuroprotective and hepatoprotective effects (13-16). In addition, several studies have indicated that salidroside is an effective antioxidant and free radical scavenger (17-19). However, the effects of salidroside on the oxidative stress induced by exhaustive exercise have not yet been elucidated. In this study, exhaustive exercise was selected as the method of inducing oxidative stress in rats and the effects of salidroside on the exhaustive exercise-induced oxidative stress in the rat liver tissue were investigated.

\section{Materials and methods}

Chemicals and reagents. Salidroside (purity 99\%) was purchased from the National Institute for the Control of Pharmaceutical and Biological Products (Beijing, China). All kits including malondialdehyde (MDA), CAT, SOD, GSH-Px and liver glycogen were purchased from the Nanjing Jiancheng 
Bioengineering Institute (Nanjing, China). All other chemicals and reagents were of analytical grade and were obtained from the usual commercial sources. Deionized water was used to prepare all solutions and in all experiments.

Animals and grouping. Male Sprague-Dawley (SD) rats (220-250 g) were purchased from the Animal Experiment Center of Tianjin University (Tianjin, China). The animals were housed at a temperature of $18-20^{\circ} \mathrm{C}$ and a humidity of 65-69\% and were submitted to a 12-h light/dark cycle. The rats had unrestricted access to tap water and standard rat chow. All procedures involving the use of laboratory animals were carried out in accordance with the National Institutes of Health guidelines. Following an adaptation period of a week, 40 animals were randomly divided into four groups of ten rats each: control (C), low-dose salidroside-treated (LT), middle-dose salidroside-treated (MT) and high-dose salidroside-treated (HT) groups. The rats in the treated groups received salidroside $(25,50$ and $100 \mathrm{mg} / \mathrm{kg}$, respectively) intragastrically (ig) and the rats in the control group received drinking water ig for 4 weeks.

Exhaustive swimming exercise. After 4 weeks, the rats performed an exhaustive swimming exercise in an acrylic plastic pool $(90 \times 45 \times 45 \mathrm{~cm})$ filled with water maintained at a temperature of $36 \pm 2^{\circ} \mathrm{C}$. The water depth, $35 \mathrm{~cm}$, was set so that the rats could not rest by supporting their tails on the bottom of the pool. Each rat had a weight attached (5\% body weight) to its tail for the duration of the swim-to-exhaustion exercise. The animals were assessed as being exhausted when they failed to rise to the surface of the water to breathe within $7 \mathrm{sec}(20,21)$. The exhaustive swimming time was recorded in min for each rat.

Biochemical parameters analysis. At the end of exhaustive swimming exercise, all rats were immediately anesthetized with ethyl ether. Liver tissue was extracted and frozen in liquid nitrogen for storage at $-80^{\circ} \mathrm{C}$ until required for MDA, CAT, SOD, GSH-Px and glycogen analysis. All biochemical parameters were tested following the recommended procedures provided in the assay kits.

Statistical analysis. The data are expressed as the mean \pm SD based on the indicated number in the experiment. All analyses of data were carried out using the Statistical Package for Social Sciences (version 11.0; SPSS, Inc., Chicago, IL, USA). The results were analyzed using one-way analysis of variance followed by a Student's t-test for comparison between various treatment groups. $\mathrm{P}<0.05$ was considered to indicate a statistically significant result.

\section{Results and Discussion}

Effect of salidroside on the exhaustive swimming time of rats. Swimming was selected as a model for exercise performance, since swimming appears to be natural behavior for rodents and humans $(22,23)$. As shown in Fig. 1, the exhaustive swimming times of the rats in the LT, MT and HT groups were significantly prolonged compared with those in the control group $(\mathrm{P}<0.05)$, and were $1.52,1.80$ and 1.96 times longer than

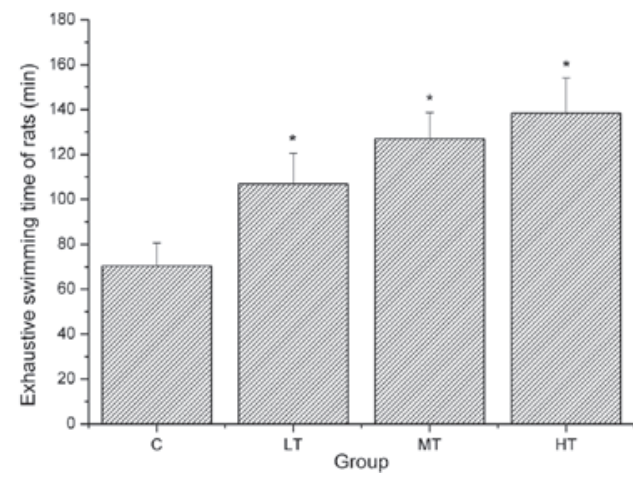

Figure 1. Effect of salidroside on the exhaustive swimming time of rats Data are the mean $\pm \mathrm{SD} .{ }^{*} \mathrm{P}<0.05$ compared with the control (C) group. LT, low-dose salidroside-treated; MT, middle-dose salidroside-treated; HT, high-dose salidroside-treated.

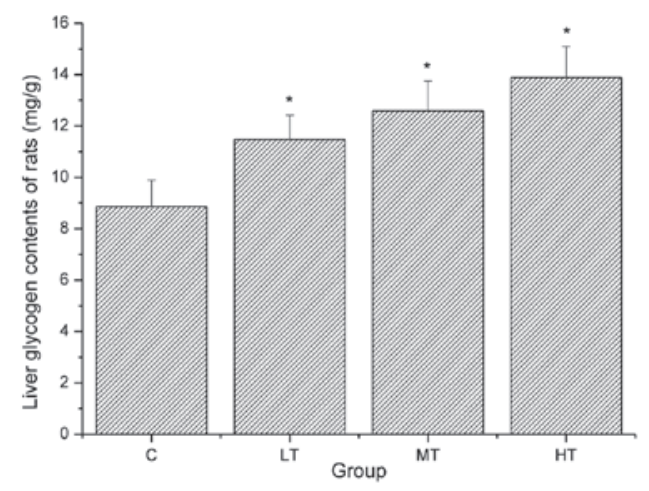

Figure 2. Effect of salidroside on the liver glycogen levels of rats. Data are the mean $\pm \mathrm{SD}$. ${ }^{*} \mathrm{P}<0.05$ compared with the control (C) group. LT, low-dose salidroside-treated; MT, middle-dose salidroside-treated; HT, high-dose salidroside-treated.

in the control group, respectively. These results suggest that salidroside is able to elevate the exercise tolerance of rats.

Effect of salidroside on the liver glycogen levels of rats. The contribution of glycogen to energy production during exhaustive exercise is necessary since glycogen may be degraded rapidly to produce ATP aerobically and anaerobically $(24,25)$. The energy for exercise is derived initially from the breakdown of glycogen and later from circulating glycogen released by the liver and from non-esterified fatty acids. Therefore, increased liver glycogen storage levels are conducive to enhancements of endurance and locomotory capacity (26). As shown in Fig. 2, the liver glycogen levels of the rats in the LT, MT and HT groups were significantly increased compared with those in the control group $(\mathrm{P}<0.05)$. These results suggest that salidroside is able to significantly increase the liver glycogen levels of the rats following exhaustive exercise by improving the glycogen reserve, reducing the glycogen consumption during exercise or both.

Effect of salidroside on the MDA levels in the liver tissues of rats. Oxidative stress induced by exhaustion exercise may produce ROS. The production of ROS is harmful to the mitochondria in the cell as the ROS affect the functioning 
Table I. Effect of salidroside on the CAT, SOD and GSH-Px levels in rat liver tissue.

\begin{tabular}{llcc}
\hline Group & $\begin{array}{c}\text { CAT } \\
(\mathrm{U} / \mathrm{mg} \cdot \text { prot})\end{array}$ & $\begin{array}{c}\text { SOD } \\
(\mathrm{U} / \mathrm{mg} \cdot \text { prot})\end{array}$ & $\begin{array}{c}\text { GSH-Px } \\
(\mathrm{U} / \mathrm{mg} \cdot \text { prot })\end{array}$ \\
\hline C & $33.49 \pm 5.15$ & $102.38 \pm 10.01$ & $317.68 \pm 42.61$ \\
LT & $39.87 \pm 4.36^{\mathrm{a}}$ & $126.39 \pm 11.47^{\mathrm{a}}$ & $389.52 \pm 46.27^{\mathrm{a}}$ \\
MT & $42.28 \pm 5.23^{\mathrm{a}}$ & $135.41 \pm 14.19^{\mathrm{a}}$ & $396.84 \pm 54.63^{\mathrm{a}}$ \\
HT & $47.62 \pm 5.94^{\mathrm{a}}$ & $137.95 \pm 12.41^{\mathrm{a}}$ & $431.19 \pm 44.34^{\mathrm{a}}$ \\
\hline
\end{tabular}

Data are mean $\pm \mathrm{SD} .{ }^{\text {a }} \mathrm{P}<0.05$ compared with the control $(\mathrm{C})$ group. $\mathrm{LT}$, low-dose salidroside-treated; MT, middle-dose salidroside-treated; HT, high-dose salidroside-treated; CAT, catalase; SOD, superoxide dismutase; GSH-Px, glutathione peroxidase.

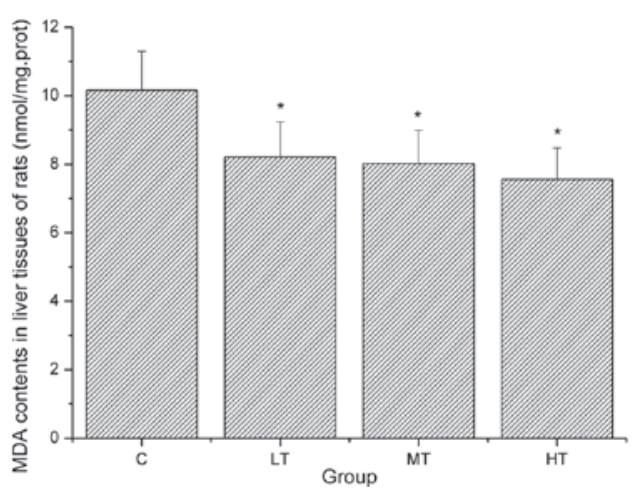

Figure 3. Effect of salidroside on the malondialdehyde (MDA) levels in the liver tissues of rats. Data are the mean $\pm \mathrm{SD}$. ${ }^{*} \mathrm{P}<0.05$ compared with the control (C) group. LT, low-dose salidroside-treated; MT, middle-dose salidroside-treated; HT, high-dose salidroside-treated.

of the mitochondria, causing them to lose their efficacy as components of the electron transport chain, which may also lead to aging or fatigue. This type of oxidization injury to the mitochondria results in lipid peroxidation and causes cell damage (27). MDA, a metabolite of phospholipid peroxidation, is a popular marker of living body oxidative damage (28). As shown in Fig. 3, the MDA levels in the liver tissues of the rats in the LT, MT and HT groups were significantly decreased compared with those in the $\mathrm{C}$ group $(\mathrm{P}<0.05)$. These results suggest that salidroside reduces lipid peroxidation.

Effect of salidroside on the CAT, SOD and GSH-Px levels in the liver tissues of rats. CAT, SOD and GSH-Px are regarded as the first line of defense of the antioxidant enzyme system against ROS generated during exhaustive exercise (29). SOD dismutates superoxide radicals to form $\mathrm{H}_{2} \mathrm{O}_{2}$ and $\mathrm{O}_{2}$. GPH-Px is an enzyme responsible for reducing $\mathrm{H}_{2} \mathrm{O}_{2}$ or organic hydroperoxides to water and alcohol, respectively. CAT catalyzes the breakdown of $\mathrm{H}_{2} \mathrm{O}_{2}$ to form water and $\mathrm{O}_{2}(30,31)$. As shown in Table I, the CAT, SOD and GSH-Px levels in the liver tissues of the rats in the LT, MT and HT groups were significantly increased compared with those in the $\mathrm{C}$ group $(\mathrm{P}<0.05)$. These results suggest that salidroside promotes increases in the activities of antioxidant enzymes (CAT, SOD and GSH-Px), which indicates that salidroside has beneficial effects on the attenuation of the oxidative stress induced by exhaustive exercise.

In conclusion, this study demonstrates that salidroside is able to elevate the exercise tolerance and increase the liver glycogen levels of rats following exhaustive exercise. Salidroside is also able to reduce MDA levels and enhance the activities of antioxidant enzymes (CAT, SOD and GSH-Px) in the liver tissues of rats. These findings indicate that salidroside is effective in preventing oxidative stress following exhaustive exercise. Therefore, salidroside may be used as an antioxidant supplement for competing athletes who participate in exhaustive endurance events.

\section{Acknowledgements}

The authors gratefully acknowledge Dr Wang Fei for help in editing the manuscript. The authors are also grateful to Dr Zhang Yang for his timely technical support.

\section{References}

1. Urso ML and Clarkson PM: Oxidative stress, exercise, and antioxidant supplementation (Review). Toxicology 189: 41-54, 2003.

2. Konrad M, Nieman DC, Henson DA, et al: The acute effect of ingesting a quercetin-based supplement on exercise-induced inflammation and immune changes in runners. Int J Sport Nutr Exerc Metab 21: 338-346, 2011.

3. Miranda-Vilela AL, Akimoto AK, Alves PC, et al: Dietary carotenoid-rich oil supplementation improves exercise-induced anisocytosis in runners: influences of haptoglobin, MnSOD (Va19Ala), CAT (21A/T) and GPX1 (Pro198Leu) gene polymorphisms in dilutional pseudoanemia (sports anemia). Genet Mol Biol 33: 359-367, 2010.

4. Neubauer O, Reichhold S, Nersesyan A, et al: Exercise-induced DNA damage: is there a relationship with inflammatory responses (Review)? Exerc Immunol Rev 14: 51-72, 2008.

5. Powers SK, Nelson WB and Hudson MB: Exercise-induced oxidative stress in humans: cause and consequences (Review). Free Radic Biol Med 51: 942-950, 2011.

6. Tanimura Y, Shimizu K, Tanabe K, et al: Exercise-induced oxidative DNA damage and lymphocytopenia in sedentary young males. Med Sci Sports Exerc 40: 1455-1462, 2008.

7. Powers SK and Jackson MJ: Exercise-induced oxidative stress: cellular mechanisms and impact on muscle force production (Review). Physiol Rev 88: 1243-1276, 2008.

8. König D, Wagner KH, Elmadfa I and Berg A: Exercise and oxidative stress: significance of antioxidants with reference to inflammatory, muscular, and systemic stress (Review). Exerc Immunol Rev 7: 108-133, 2001.

9. Williams MH: Dietary supplements and sports performance: introduction and vitamins. J Int Soc Sports Nutr 1: 1-6, 2004.

10. Sen CK: Antioxidants in exercise nutrition (Review). Sports Med 31: 891-908, 2001

11. Kelly GS: Rhodiola rosea: a possible plant adaptogen (Review). Altern Med Rev 6:293-302, 2001.

12. Li HB and Chen F: Preparative isolation and purification of salidroside from the Chinese medicinal plant Rhodiola sachalinensis by high-speed counter-current chromatography. J Chromatogr A 932: 91-95, 2001

13. Li F, Tang H, Xiao F, et al: Protective effect of salidroside from Rhodiolae Radix on diabetes-induced oxidative stress in mice. Molecules 16: 9912-9924, 2011.

14. Mao GX, Deng HB, Yuan LG, et al: Protective role of salidroside against aging in a mouse model induced by D-galactose. Biomed Environ Sci 23: 161-166, 2010.

15. Wu YL, Lian LH, Jiang YZ and Nan JX: Hepatoprotective effects of salidroside on fulminant hepatic failure induced by D-galactosamine and lipopolysaccharide in mice. J Pharm Pharmacol 61: 1375-1382, 2009.

16. Wang H, Ding Y, Zhou J, et al: The in vitro and in vivo antiviral effects of salidroside from Rhodiola rosea $\mathrm{L}$. against coxsackievirus B3. Phytomedicine 16: 146-155, 2009. 
17. Yu P, Hu C, Meehan EJ and Chen L: X-ray crystal structure and antioxidant activity of salidroside, a phenylethanoid glycoside. Chem Biodivers 4: 508-513, 2007.

18. Zhang Y and Liu Y: Study on effects of salidroside on lipid peroxidation on oxidative stress in rat hepatic stellate cells. Zhong Yao Cai 28: 794-796, 2005 (In Chinese).

19. De Sanctis R, De Bellis R, Scesa C, et al: In vitro protective effect of Rhodiola rosea extract against hypochlorous acidinduced oxidative damage in human erythrocytes. Biofactors 20 : 147-159, 2004.

20. Oh TW, Oh TW and Ohta F: Dose-dependent effect of capsaicin on endurance capacity in rats. Br J Nutr 90: 515-520, 2003.

21. Misra DS, Maiti R and Ghosh D: Protection of swimming-induced oxidative stress in some vital organs by the treatment of composite extract of Withania somnifera, Ocimum sanctum and Zingiber officinalis in male rat. Afr J Tradit Complement Altern Med 6: 534-543, 2009.

22. Burneiko RC, Diniz YS, Galhardi CM, et al: Interaction of hypercaloric diet and physical exercise on lipid profile, oxidative stress and antioxidant defenses. Food Chem Toxicol 44: 1167-1172, 2006.

23. Kramer K, Dijkstra H and Bast A: Control of physical exercise of rats in a swimming basin. Physiol Behav 53: 271-276, 1993.
24. Fallowfield JL and Williams C: Carbohydrate intake and recovery from prolonged exercise. Int J Sport Nutr 3: 150-164, 1993.

25. Nicholas CW, Green PA, Hawkins RD and Williams C: Carbohydrate intake and recovery of intermittent running capacity. Int J Sport Nutr 7: 251-260, 1997.

26. Tang W, Zhang Y, Gao J, et al: The anti-fatigue effect of 20(R)-ginsenoside $\operatorname{Rg} 3$ in mice by intranasally administration. Biol Pharm Bull 31: 2024-2027, 2008.

27. Wang JJ, Shieh MJ, Kuo SL, et al: Effect of red mold rice on antifatigue and exercise-related changes in lipid peroxidation in endurance exercise. Appl Microbiol Biotechnol 70: 247-253, 2006.

28. Lu HK, Hsieh CC, Hsu JJ, et al: Preventive effects of Spirulina platensis on skeletal muscle damage under exercise-induced oxidative stress. Eur J Appl Physiol 98: 220-226, 2006.

29. Huang CC, Lin TJ, Lu YF, et al: Protective effects of L-arginine supplementation against exhaustive exercise-induced oxidative stress in young rat tissues. Chin J Physiol 52: 306-315, 2009.

30. You LJ, Zhao MM, Regenstein JM and Ren JY: In vitro antioxidant activity and in vivo anti-fatigue effect of loach (Misgurnus anguillicaudatus) peptides prepared by papain digestion. Food Chem 124: 188-194, 2011.

31. Jin HM and Wei P: Anti-fatigue properties of tartary buckwheat extracts in mice. Int J Mol Sci 12: 4770-4780, 2011. 